\title{
Differential effects of IRS1 phosphorylated on Ser307 or Ser632 in the induction of insulin resistance by oxidative stress
}

\author{
A. Bloch-Damti • R. Potashnik • P. Gual • \\ Y. Le Marchand-Brustel • J. F. Tanti • A. Rudich • \\ N. Bashan
}

Received: 20 April 2006 / Accepted: 10 May 2006/ Published online: 3 August 2006

(C) Springer-Verlag 2006

\begin{abstract}
Aims/hypothesis Induction of stress kinases leading to serine hyperphosphorylation of IRS1 may link oxidative stress to insulin resistance. The aim of this study was to investigate the roles of the phosphorylated serine residues Ser307 and Ser632, two sites implicated in the inhibition of IRS1 function in insulin signalling.

Materials and methods Fao hepatoma cells were exposed to an $\mathrm{H}_{2} \mathrm{O}_{2}$-generating system, and antibodies against the two phosphorylated serine residues were used for immunoprecipitation, immunoblot and immunofluorescence analyses.

Results Exposure to $\sim 50 \mu \mathrm{mol} / \mathrm{L}_{2} \mathrm{O}_{2}$ for $2 \mathrm{~h}$ resulted in IRS1 phosphorylation on both Ser307 and Ser632, concomitant with activation of inhibitor kappa kinase $\beta$ (IKK $\beta)$ and c-Jun kinase (JNK). Immunoprecipitation studies revealed that the maximum overlap between phospho (p) Ser307IRS1 and pSer632-IRS1 was 20\%, and confocal microscopy suggested distinct localisations of IRS1 molecules phosphorylated on either site. Although pSer307-IRS1 showed
\end{abstract}

\footnotetext{
A. Bloch-Damti $\cdot$ R. Potashnik $\cdot$ A. Rudich $\cdot$ N. Bashan $(\square)$ Department of Clinical Biochemistry, Faculty of Health Sciences, Ben-Gurion University of the Negev,

Beer-Sheva 84103, Israel

e-mail: nava@bgu.ac.il

P. Gual $\cdot$ Y. Le Marchand-Brustel · J. F. Tanti

INSERM, U568,

Nice F-06107, France

P. Gual · Y. Le Marchand-Brustel · J. F. Tanti

Faculté de Medecine, IFR50, University of Nice Sophia Antipolis,

Nice F-06107, France

\section{A. Rudich}

The S. Daniel Abraham Center for Health and Nutrition,

Ben-Gurion University of the Negev,

Beer-Sheva 84103, Israel
}

decreased insulin-induced tyrosine phosphorylation and interaction with phosphatidylinositol 3-kinase (PI3K) in response to insulin, pSer632-IRS1 molecules were normally tyrosine-phosphorylated and exhibited typical associated PI3K activity. Salicylic acid and SP600125 partially inhibited IKK $\beta$ and JNK, respectively, which indicated distinct roles for these two kinases in the phosphorylation of IRS1 at the two serine sites. Protection against oxidationmediated impairment in insulin-induced phosphorylation of protein kinase $\mathrm{B} / \mathrm{Akt}$ and in glycogen synthesis was achieved only by combining salicylic acid and SP600125.

Conclusions/interpretation These results suggest that pSer307-IRS1 and pSer632-IRS1 may define two minimally overlapping pools of IRS1 in response to oxidative stress, contributing differentially to insulin resistance. A combination of stress kinase inhibitors is required to protect against insulin resistance and IRS1 hyperphosphorylation induced by oxidative stress.

Keywords c-Jun kinase - Glycogen synthesis ·

Hydrogen peroxide $\cdot \operatorname{IKK} \beta \cdot$ Inhibitor kappa kinase $\beta$.

Stress kinases

$\begin{array}{ll}\text { Abbreviations } \\ \text { IKK } \beta \quad \text { inhibitor of kappa B kinase } \beta \\ \text { JNK } & \text { c-Jun kinase } \\ \text { PI3K } & \text { phosphatidylinositol 3-kinase } \\ \text { PKB } & \text { protein kinase B }\end{array}$

\section{Introduction}

The diabetic and obese states are increasingly being shown to induce stress responses in tissues relevant to metabolic 
regulation. Stress signalling cascades are signalling pathways involving serine/threonine 'stress kinases', which may be activated in response to diverse conditions and cues, including inflammatory cytokines and endoplasmic reticulum stress, both of which are implicated in the pathogenesis of insulin resistance. Once activated, stress kinases can impinge upon the insulin signalling cascade, leading to insulin resistance $[1,2]$. One mechanism by which this may occur is through stress kinase-induced serine/threonine phosphorylation of the insulin receptor substrate (IRS) proteins [3-5]. Specifically, IRS1 Ser307 has been shown to be a site phosphorylated by several kinases, including mTOR [6-9], inhibitor of kappa B kinase $\beta$ (IKK $\beta)[10,11]$ and c-Jun kinase (JNK) [9, 12-14]. This rendered IRS1 molecules phosphorylated on this site less capable of becoming tyrosine-phosphorylated in response to insulin, hence having decreased capacity to interact with phosphatidylinositol 3-kinase (PI3K). Elevated levels of phospho (p)-Ser307-IRS1 have been reported in animal models of insulin resistance [15], and the equivalent Ser312 site in human IRS1 has been demonstrated to be hyperphosphorylated in insulin-resistant subjects [16]. Phosphorylation of IRS1 on Ser632 has also been implicated in insulin resistance. Hyperosmolar stress induced pSer632 hyperphosphorylation [7], and an increase in the phosphorylation state of the human equivalent Ser636 was present in myotubes from patients with type 2 diabetes [17], their insulin-resistant offspring [16] and in healthy subjects infused with TNF- $\alpha$ [18].

The wealth of information suggesting a role for serine/ threonine hyperphosphorylation of IRS molecules in the induction of insulin resistance suggests that inhibition of the responsible kinases may constitute a viable approach to the relief of insulin resistance. This approach is particularly attractive for the stress kinases, which are not traditionally viewed as intrinsic components of the insulin signalling cascade. Hence, their inhibition is unlikely to impair normal signal propagation. Defining which stress kinase inhibitor(s) could prevent insulin resistance, and in response to which type of physiologically relevant inducers, is of interest, as it sheds light both on basic mechanisms leading to insulin resistance and on potential therapeutic interventions.

Oxidative stress is increasingly implicated as a common mechanism mediating the pathogenesis of insulin resistance in response to potential inducers, including inflammatory cytokines, endoplasmic reticulum stress and nutrient access $[1,19,20]$. Evidence exists for increased systemic oxidative stress and for oxidative stress at the adipose tissue level of obese persons $[2,21]$ and in the livers of persons with hepatic steatosis [22], a close surrogate of insulin resistance. Using cellular model systems, it has been shown by others and us that oxidative stress impairs insulin action [23-26], associated with serine phosphorylation of IRS1
[27]. Intriguingly, overall serine/threonine hyperphosphorylation did not correlate with the degree of insulin resistance at the metabolic level [27]. In parallel, oxidative stress is known to activate several stress signalling cascades, including $\mathrm{JNK}$ and the IKK $\beta$-nuclear factor kappa B signalling cascades [1]. Here we examined: (i) the role of these kinases in the induction of insulin resistance and IRS1 phosphorylation of Ser307 and Ser632 by oxidative stress; (ii) the significance of phosphorylation of these specific residues for IRS1 function; and (iii) whether insulin resistance can be relieved by pharmacological inhibitors of these stress kinases.

\section{Materials and methods}

\section{Reagents}

Tissue culture medium, serum and antibiotic solutions were obtained from Biological Industries (Beit-Haeemek, Israel). Recombinant human insulin was from Novo Nordisk (Bagsvaerd, Denmark). Polyclonal IRS1 antibodies (Cterminus) and anti-p85 were purchased from Upstate Biotechnology, Inc. (Lake Placid, NY, USA). Polyclonal pSer307-IRS1 and pSer632-IRS1 were generated as described previously [6]. Polyclonal phospho-c-Jun, monoclonal p-JNK, polyclonal JNK1/2 and monoclonal IKK $\beta$ antibodies were purchased from Santa Cruz Biotechnologies (Santa Cruz, CA, USA). Polyclonal p-IkB and pSer473-protein kinase B (PKB)/Akt antibodies were purchased from Cell Signaling (Danvers, MA, USA). SP600125, a reversible ATP-competitive inhibitor of JNK, was purchased from Biomol (Plymouth Meeting, PA, USA). Peroxidase-conjugated anti-rabbit IgG, anti-mouse $\operatorname{IgG}$ and $\left[\gamma_{-}{ }^{32} \mathrm{P}\right] \mathrm{ATP}$ were from Amersham Life Sciences (Little Chalfont, Bucks, UK). Protein A-Sepharose was from Pharmacia Biotech (Uppsala, Sweden). U- ${ }^{14}$ C-glucose was purchased from Nuclear Research Center Negev (Dimona, Israel). Salicylate and all other chemicals were obtained from Sigma Chemical Co. (St Louis, MO, USA).

\section{Cell culture and treatment}

Rat hepatoma Fao cells were grown to confluence in RPMI medium containing $5 \mathrm{mmol} / \mathrm{l}$ glucose with $10 \%$ foetal calf serum. Before experiments, cells were deprived of serum for $16 \mathrm{~h}$ by incubation in medium supplemented with $0.5 \%$ $(w / v)$ radioimmunoassay grade BSA. Cells were then rinsed three times in PBS, exposed to fresh medium with or without glucose oxidase [24, 28], and rinsed again before stimulation with $100 \mathrm{nmol} / \mathrm{l}$ insulin. The amount of $\mathrm{H}_{2} \mathrm{O}_{2}$ in the medium was measured spectrophotometrically as described previously [29]. 
Glycogen synthesis

Incorporation of $\mathrm{U}^{14}{ }^{14} \mathrm{C}$-glucose into glycogen in Fao cells was measured as described previously [24, 27].

Whole-cell lysates, post-nuclear internal membrane preparations and western blots

After treatment, cells were thoroughly washed with icecold PBS and scraped into $0.2 \mathrm{ml}$ of ice-cold lysis buffer $(50 \mathrm{mmol} / 1$ Tris- $\mathrm{HCl}, 1 \%$ Nonidet $\mathrm{P}-40,0.25 \%$ sodium deoxycholate, $150 \mathrm{mmol} / 1 \mathrm{NaCl}, 1 \mathrm{mmol} / \mathrm{l}$ EGTA, $1 \mathrm{mmol} / 1$ sodium vanadate, $1 \mathrm{mmol} / \mathrm{l} \mathrm{NaF}$ and protease inhibitors [1:1,000 dilution of protease inhibitor cocktail Sigma P-8340]). Lysates were shaken gently for $15 \mathrm{~min}$ at $4^{\circ} \mathrm{C}$ and centrifuged $\left(12,000 \times g, 15 \mathrm{~min}\right.$ at $\left.4^{\circ} \mathrm{C}\right)$, the supernatant was collected, and protein content was determined (BCA method; Pierce, Rockford, IL, USA). Postnuclear internal membrane fractions were prepared from three $10-\mathrm{cm}$ dishes of confluent Fao cells per treatment. Cells were homogenised in $2.5 \mathrm{ml}$ fractionation buffer (255 mmol/1 sucrose, $20 \mathrm{mmol} / \mathrm{l}$ HEPES, pH 7.4, $1 \mathrm{mmol} / 1 \mathrm{EDTA}, 0.2 \mathrm{mmol} / 1$ sodium vanadate with a 1:1,000 dilution of protease inhibitor cocktail). Nuclei and debris were sedimented by centrifugation at $1,000 \times g$ for $5 \mathrm{~min}$, after which the supernatants were subjected to two rounds of centrifugation $(30,000 \times g$ for $30 \mathrm{~min}$ then $250,000 \times \mathrm{g}$ for $90 \mathrm{~min})$. Pellets were resuspended in fractionation buffer to $5 \mathrm{mg}$ of protein $/ \mathrm{ml}$. Laemmli buffer was added and samples were boiled for $5 \mathrm{~min}$. Protein samples were resolved by $7.5-10 \%$ SDS-PAGE and subjected to western blotting, followed by quantitation using video densitometry analysis, as described previously $[28,30]$.

Immunoprecipitation and PI3K assay

Cells were lysed in ice-cold deoxycholate based buffer, as described above. For immunoprecipitation, $0.5 \mathrm{mg}$ protein of cell lysate was used, and the PI3K assay was performed following the protocol described by Hadari et al. [31] using phosphatidylinositol and $\left[\gamma_{-}{ }^{32} \mathrm{P}\right]$ ATP as substrates, as reported previously $[28,30]$. The phosphorylated phosphatidylinositols were separated using thin-layer chromatography and quantified by video densitometry.

\section{Fluorescence microscopy}

After cell treatments, Fao cells grown on $15-\mathrm{mm}$ diameter glass coverslips were fixed with $4 \%$ (v/v) paraformaldehyde in PBS for $25 \mathrm{~min}$ (first $5 \mathrm{~min}$ on ice) then rinsed, and excess paraformaldehyde was quenched with $100 \mathrm{mmol} / \mathrm{l}$ glycine for $10 \mathrm{~min}$. Cells were then permeabilised with $0.1 \%(\mathrm{v} / \mathrm{v})$ Triton X-100 in PBS for 20 min and blocked for 30 min with
PBS containing $5 \%$ goat serum $(\mathrm{v} / \mathrm{v})$, as described previously [32]. The following dilutions in blocking buffer of the primary antibodies were used: anti-IRS1, 1:100; antipSer307-IRS1, 1:150; anti-pSer632-IRS1, 1:150. After $1 \mathrm{~h}$ of incubation at room temperature, cells were rinsed with PBS and incubated for an additional $1 \mathrm{~h}$ in the dark with Alexa555-conjugated anti-rabbit IgG secondary antibody at a dilution of 1:750. Cell monolayers were washed further with PBS and mounted onto glass slides using fluorescent mounting medium (DakoCytomation, Carpinteria, CA, USA). For confocal fluorescence microscopy, cells were examined with a Zeiss LSM 510 laser scanning confocal microscope with $\times 40$ and $\times 100$ oil immersion objectives. Acquisition parameters were adjusted to exclude saturation of the pixels, and to exclude background fluorescence determined by incubating cells only with secondary antibody.

\section{Statistical analysis}

Data are expressed as mean \pm SEM. Each treatment was compared with a control, and differences between pairs of groups were evaluated using Student's $t$-test. The criterion for significance was set at $p<0.05$. When multiple groups were compared, ANOVA was used for analysis.

\section{Results}

IRS1 phosphorylated on Ser307 or Ser632 in response to oxidative stress represents seemingly distinct pools of IRS1 protein

The Fao hepatoma cell line was used as a cellular model of liver cells, as Fao cells respond acutely to insulin stimulation with a metabolically relevant increase in glycogen synthesis. Cells were exposed to oxidative stress by $2 \mathrm{~h}$ of incubation in the presence of glucose oxidase, continuously generating submillimolar $\mathrm{H}_{2} \mathrm{O}_{2}$ concentrations (see Materials and methods for details), as we have described previously [24, 28]. Insulin-stimulated glycogen synthesis (Fig. 1a) was markedly impaired after exposure to the $\mathrm{H}_{2} \mathrm{O}_{2}$-generating system. Insulin-stimulated activation of $\mathrm{PKB} / \mathrm{Akt}$ is thought to participate in mediating insulin's action on glycogen synthesis, and, consistently with the metabolic insulin resistance, oxidative stress impaired insulin-stimulated Ser473 phosphorylation of PKB/Akt (Fig. 1b). This signalling effect of oxidative stress seen in Fao cells was consistent with our previous observations in 3T3-L1 adipocytes [30, 33].

Serine hyperphosphorylation of IRS1 was observed in a time-dependent manner in response to oxidative stress, as indicated by retarded migration of the IRS1 band (Fig. 2a, upper blot). This effect was associated at $2 \mathrm{~h}$ of exposure with a decrease of up to $20 \%$ in total IRS1 content, which 
$\mathbf{a}$

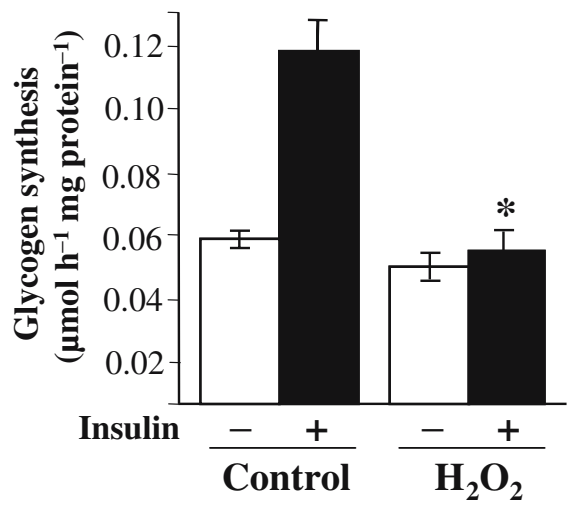

Fig. 1 Oxidative stress induces resistance to the acute metabolic and signalling effects of insulin. a Fao hepatoma cells were starved of serum overnight then incubated for $2 \mathrm{~h}$ in the absence or presence of $50 \mu \mathrm{mol} / 1 \quad \mathrm{H}_{2} \mathrm{O}_{2}$ generated by the addition of glucose oxidase $(100 \mathrm{mU} / \mathrm{ml})$ to the glucose-containing medium. Cells were rinsed, and then stimulated with or without $100 \mathrm{nmol} / 1$ insulin for $60 \mathrm{~min}$, during which incorporation of glucose into glycogen was measured as described in Materials and methods. Results are mean \pm SEM of at b

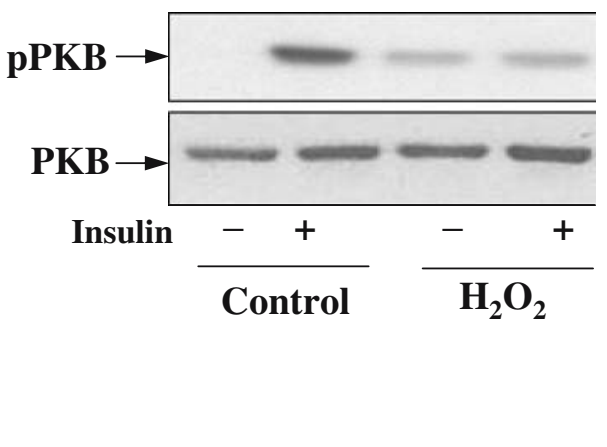

least four independent experiments performed in duplicate. ${ }^{*} p<0.05$ vs insulin-stimulated control cells. b PKB/Akt Ser473 phosphorylation was measured after exposure to oxidative stress as described above, without or with insulin stimulation for $10 \mathrm{~min}$. Lysates were prepared and western blot analysis was performed using anti-pSer473-PKB/Akt or anti-PKB/Akt antibodies. Blots representative of six independent experiments are shown

Serine phosphorylation of IRS1 was previously suggested to regulate the cellular localisation of IRS proteins and to subject hyperphosphorylated IRS1 molecules to enhanced protein degradation. To assess whether the small overlap between pSer632-IRS1 and pSer307-IRS1 is reflected in differential cellular localisation, we used indirect immunofluorescence. Figure 3 demonstrates the immunofluorescence findings, which are qualitatively consistent with the western blot analysis shown in Fig. 2. Total IRS1 protein tended to be decreased in oxidised cells (compare panels b and a in Fig. 3), as we have described previously [27]. However, the amount of both pSer632-IRS1 and pSer307-IRS1 was increased (Fig. 3; compare panel $\mathrm{d}$ with panel $\mathrm{c}$ and panel $\mathrm{f}$ with panel e, respectively). Using higher magnification, total IRS1 protein exhibited diffuse cytosolic and punctate staining (Fig. 3g). Remarkably, whereas pSer307-IRS1 exhibited more pronounced perinuclear staining, pSer632-IRS1 was more discernible in the cell periphery (Fig. 3i and h, respectively).

An acute effect of insulin has been shown to relocalise IRS1 from the membrane fraction to the cytosol ([30, 34, 35] and Fig. 4a, upper blot). Interestingly, in response to oxidative stress, pSer632-IRS1 displayed a similar effect, whereas pSer307-IRS1 failed to dissociate from a postnuclear internal membrane preparation following acute insulin stimulation (Fig. 4a, middle and lower blots, respectively). Combined, these data suggest that the two pSer sites of IRS1 may define spatially distinct pools of IRS1 proteins in response to oxidative stress. 
a

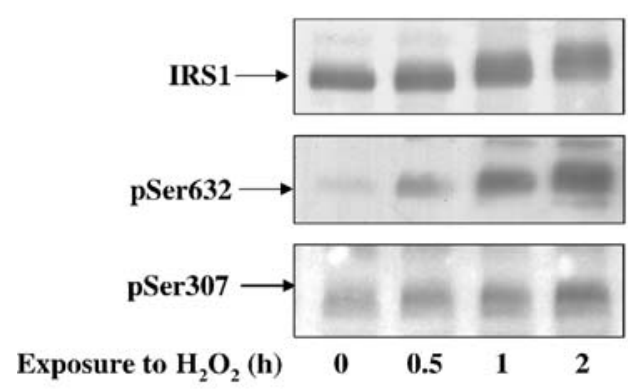

c

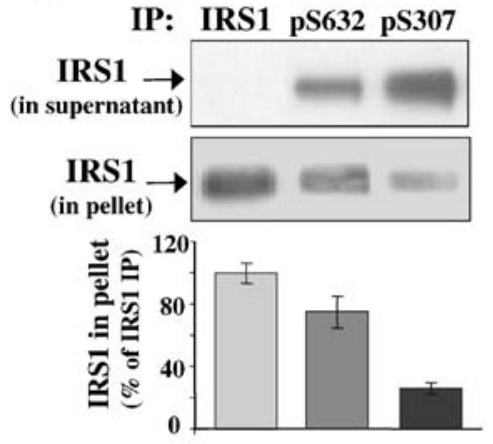

b
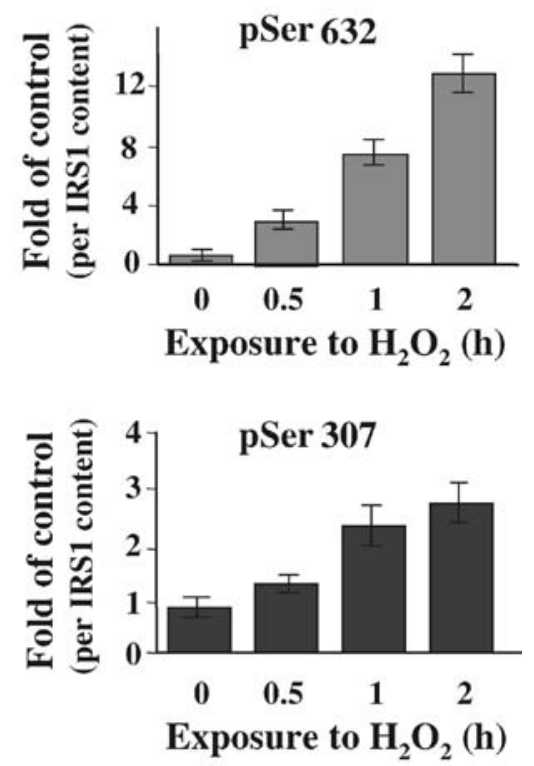

d

\section{IP: pS632 pS307}

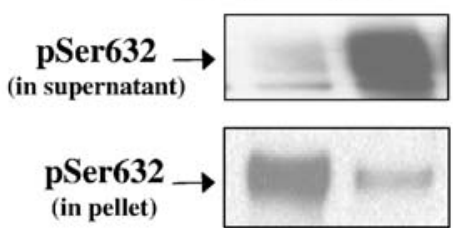

anti-IRS1, anti-pSer307-IRS1 or anti-pSer632-IRS1 antibodies. Supernatants (upper blot) or immunoprecipitates (lower blots) were subjected to western blot analysis using anti-IRS1 antibodies. Representative blots are shown, and below the blots are indicated the mean \pm SEM densitometry values derived from three independent experiments. d Supernatants (upper) or immunoprecipitates (lower) of pSer632-IRS1 and pSer307-IRS1 were subjected to immunoblotting using anti-pSer632 antibodies. This experiment was performed twice with similar results

impaired activation of PI3K was observed in a specific cellular compartment, the low-density microsomal fraction, potentially explaining the defect in downstream signal propagation to $\mathrm{PKB} / \mathrm{Akt}$ [30]. Total IRS1 proteins, pSer632-IRS1 or pSer307-IRS1 proteins were immunoprecipitated using the respective antibodies, and in each immunoprecipitate we determined IRS1 protein, its tyrosine phosphorylation, interaction with the p 85 regulatory subunit of PI3K, and IRS1-associated PI3K activity (Fig. 4b). As shown in the upper panel, pSer307-IRS1 represented a small fraction of total IRS1 protein in oxidised cells (similar to the data shown in Fig. 2c). Nevertheless, insulinstimulated tyrosine phosphorylation of pSer307-IRS1, its interaction with $\mathrm{p} 85$, and the associated PI3K activity were markedly decreased. In contrast, pSer632-IRS1 molecules displayed a high capacity to undergo insulin-induced 


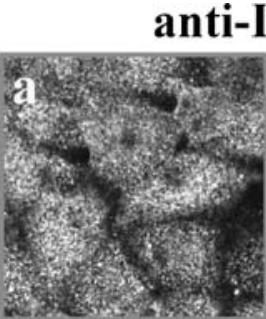

Control

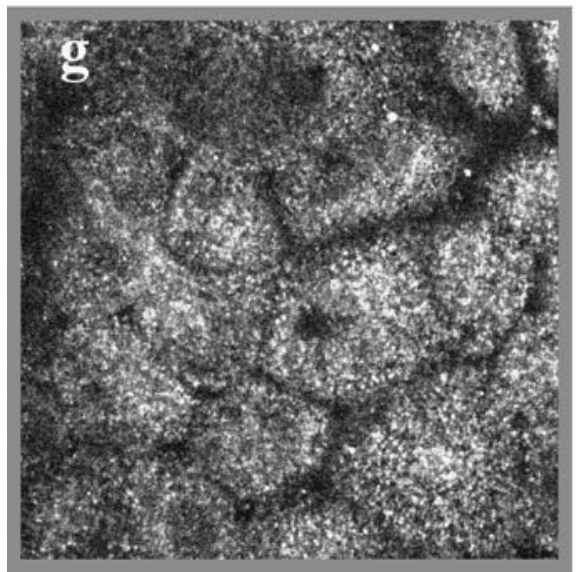

anti-pSer632-IRS1

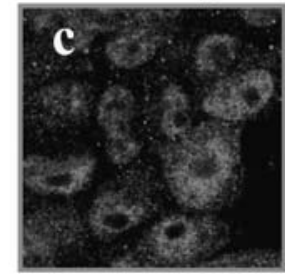

Control
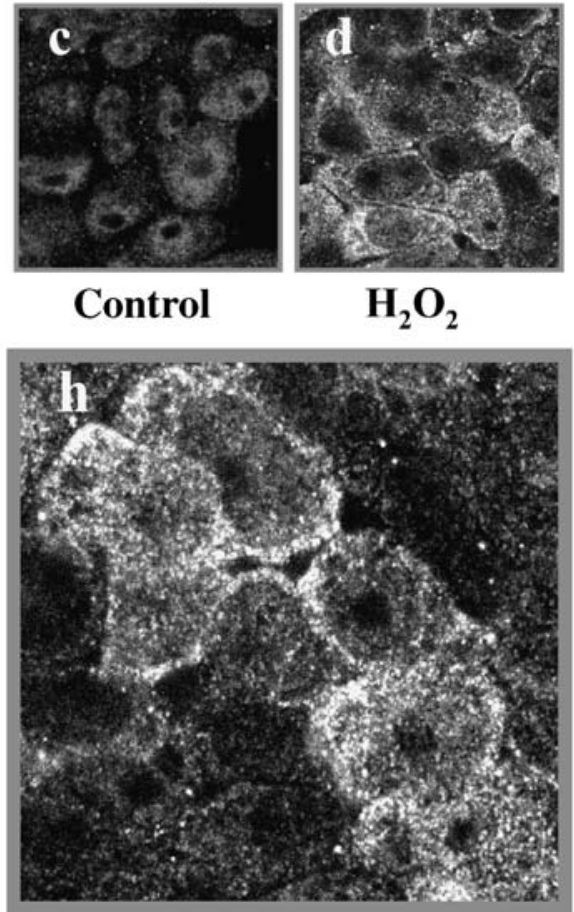

$\mathrm{H}_{2} \mathrm{O}_{2}$
anti-pSer307-IRS1

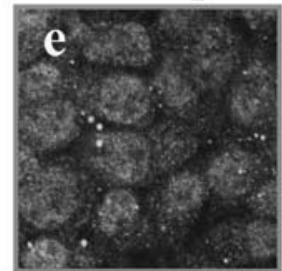

Control

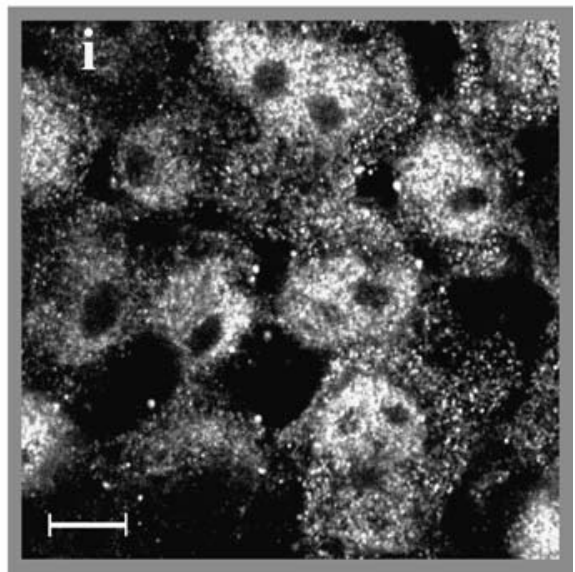

Fig. 3 Differential cellular localisation of pSer307-IRS1 and pSer632-IRS1 in oxidised Fao cells revealed by indirect immunofluorescence. Fao cells were treated as described above, fixed, permeabilised and immunostained for IRS1 $(\mathbf{a}, \mathbf{b}, \mathbf{g})$, pSer632-IRS1 (c, d, h) or pSer307-IRS1 (e, f, i), followed by laser confocal microscopy analysis as described in Materials and methods. Repre- sentative images from three independent experiments are shown of a single focal plane set to the middle height of the cells (in the $z$-axis). Sections $\mathbf{g}, \mathbf{h}$ and $\mathbf{i}$ are enlarged images of IRS1, pSer632-IRS1 and pSer307-IRS1 immunofluorescence, respectively, after exposure of the cells to oxidative stress. Scale bar $=10 \mu \mathrm{m}$

amount of IKK $\beta$ or of JNK $1 / 2$ was unchanged by up to $2 \mathrm{~h}$ of exposure to oxidative stress (lower panels of Fig. 5a and $\mathrm{b}$, respectively). Oxidative stress-induced activation of IKK $\beta$, assessed as the degree of phosphorylation of its substrate, IKB, revealed a gradual effect over time (Fig. 5a, upper blot). Similarly, JNK was activated, as indicated by the increase in its phosphorylated form, but the effect was maximal after $1 \mathrm{~h}$ of exposure to $\mathrm{H}_{2} \mathrm{O}_{2}$ (Fig. 5b, upper blot). Figure $5 \mathrm{c}$ demonstrates in a quantitative manner the dynamic of oxidative stress-induced activation of JNK and $\operatorname{IKK} \beta$, revealing that both kinases are activated in a timedependent manner which largely coincides with the increase in Ser307 and Ser632 phosphorylation of IRS1 (Fig. 2a,b).

To examine the role of each of these kinases in IRS1 phosphorylation, we used established pharmacological inhibitors. Using antibodies against the JNK substrate phospho-c-Jun and the IKK $\beta$ substrate phospho-IKB, 48\% inhibition of JNK and $65 \%$ diminution of IKK $\beta$ activity was observed with SP600125 and salicylic acid, respectively (Fig. 6a). Using these inhibitors, the contributions of IKK $\beta$ and JNK1/2 to IRS1 phosphorylation on Ser307 and Ser632 in response to oxidative stress were estimated. IRS1 phosphorylation on Ser307 was significantly inhibited by salicylic acid (Fig. 6b), probably reflecting a role for IKK $\beta$. 
a

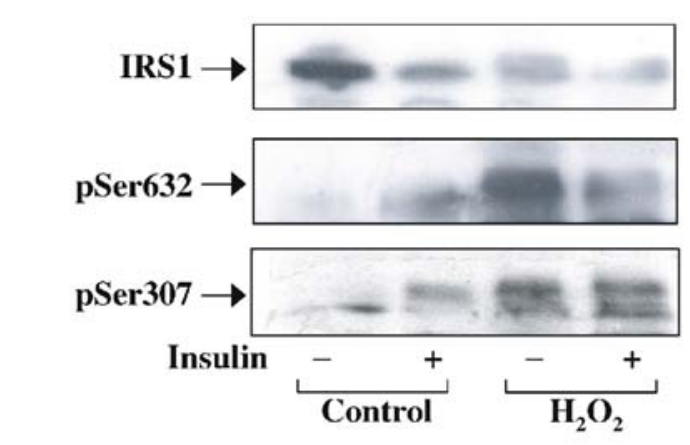

b

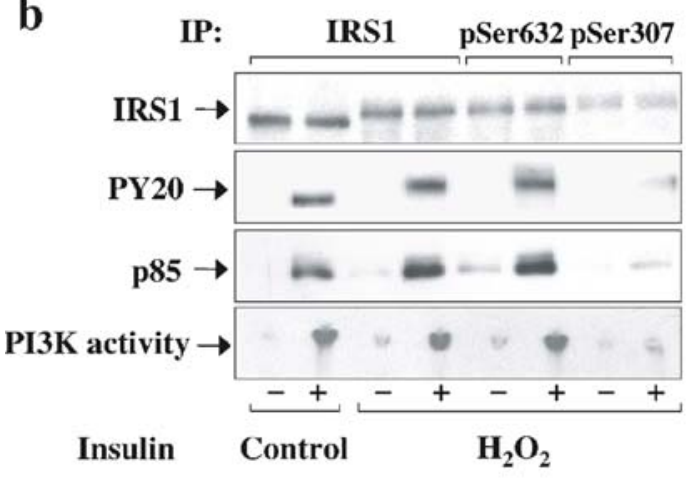

c
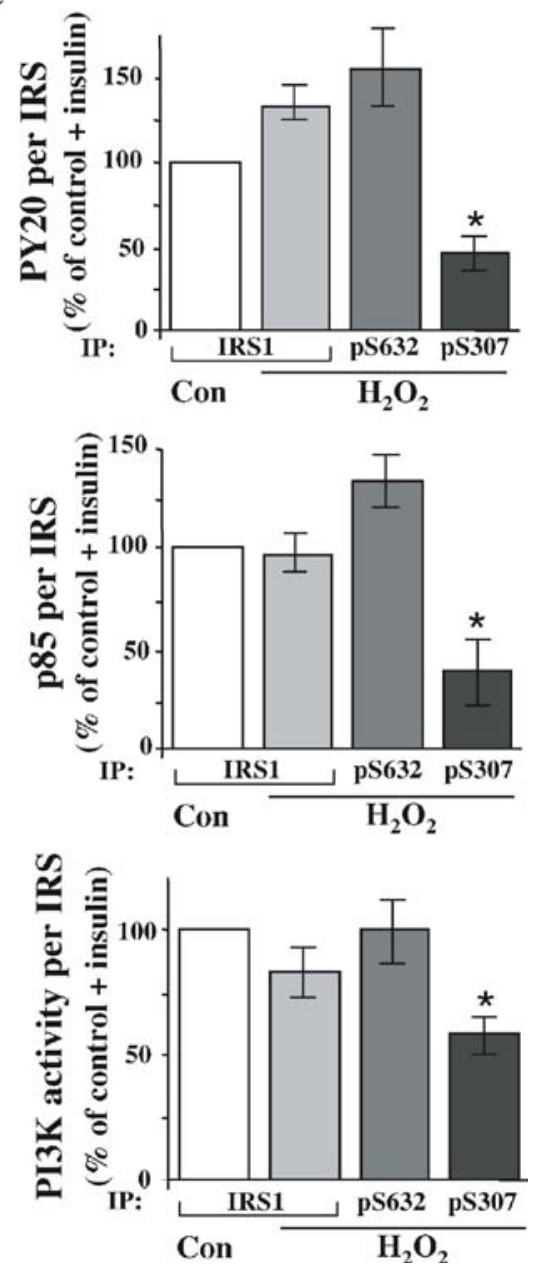

Fig. 4 IRS1 molecules phosphorylated on Ser307 but not on Ser632 exhibit impaired insulin-induced tyrosine phosphorylation, interaction with PI3K and release from internal membranes a Postnuclear internal membrane fractions were prepared as described in Materials and methods from control or oxidised cells stimulated or not stimulated with $100 \mathrm{nmol} / \mathrm{l}$ insulin for $5 \mathrm{~min}$. Equal protein contents from each preparation were resolved by SDS-PAGE and assessed for the amount of IRS1, pSer632-IRS1 or pSer307-IRS1 using the respective antibodies. This experiment was repeated twice with similar results. b Fao cells were treated as above before cell lysates were prepared, and $500 \mu \mathrm{g}$ protein was subjected to immunoprecipitation using anti-IRS1, anti-pSer632-IRS1 or anti-pSer307-IRS1. Immunoprecipitates were

Importantly, salicylic acid did not fully inhibit oxidative stress-induced IRS1 Ser307 phosphorylation, indicating incomplete inhibition of IKK $\beta$ (as suggested in Fig. 6a) and/or involvement of other, salicylic acid-insensitive serine kinases. Consistent with this notion was the finding that a decrease of nearly $20 \%$ in oxidation-induced Ser307 phosphorylation of IRS1was observed with LY294002, rapamycin or PD98059 (data not shown). Surprisingly, SP600125 had minimal effect on IRS1 phosphorylation on Ser307, suggesting incomplete inhibition of JNK or lack of involvement of this kinase in Ser307 phosphorylation in subjected to immunoblotting using anti-IRS1, anti-phosphotyrosine (PY20) antibodies, or antibodies directed against the regulatory subunit of PI3K (p85). Alternatively, PI3K activity was measured in immunoprecipitates (bottom panel), as described in Materials and methods. Blots shown are representative of three independent experiments yielding similar results. c Densitometry analysis of the ratio between the phosphotyrosine, p85 bands, or the PI3P generated in the PI3K assay and the respective IRS1 band. Densitometry values from three independent experiments were used; $100 \%$ was assigned to the ratio in IRS1 immunoprecipitate from insulin-stimulated control cells. ${ }^{*} p<0.05$ vs IRS1 immunoprecipitates from oxidised cells. Con control cells

response to oxidative stress. Consistently, the combination of salicylic acid with SP600125 was without significant additional inhibitory effect on Ser307 phosphorylation compared with salicylic acid alone (Fig. 6b).

The sensitivity to salicylic acid of Ser632 phosphorylation of IRS1 was similar to that of Ser307 phosphorylation (Fig. 6c), suggesting a contribution of a salicylic acidsensitive kinase, potentially IKK $\beta$. However, in contrast to Ser307 phosphorylation, the phosphorylation of the Ser632 site was highly sensitive to SP600125. Furthermore, the combined use of salicylic acid and SP600125 inhibited IRS1 
Fig. 5 Activation of IKK $\beta$ and JNK by oxidative stress. Fao cells were exposed to $\mathrm{H}_{2} \mathrm{O}_{2}$ generated by glucose oxidase for $0.5-2 \mathrm{~h}$, after which cells were washed, lysed and subjected to western blot analysis using antiphospho-IkappaB (pIkB), antiIKK $\beta$, anti-p-JNK $1 / 2$ or anti-JNK1/2. a, b Representative blots. c Results of densitometry analysis of the phosphoprotein bands over time presented as percentage of maximal intensity. These experiments were repeated twice with identical results. Filled squares, p-JNK; empty circles, $\mathrm{p}-\mathrm{I} \kappa \mathrm{B}$ a

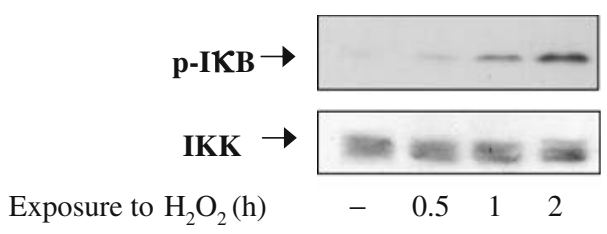

b

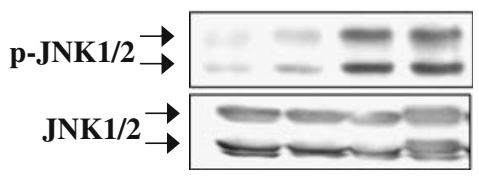

Exposure to $\mathrm{H}_{2} \mathrm{O}_{2}(\mathrm{~h}) \quad-\quad \begin{array}{llll}0.5 & 1 & 2\end{array}$

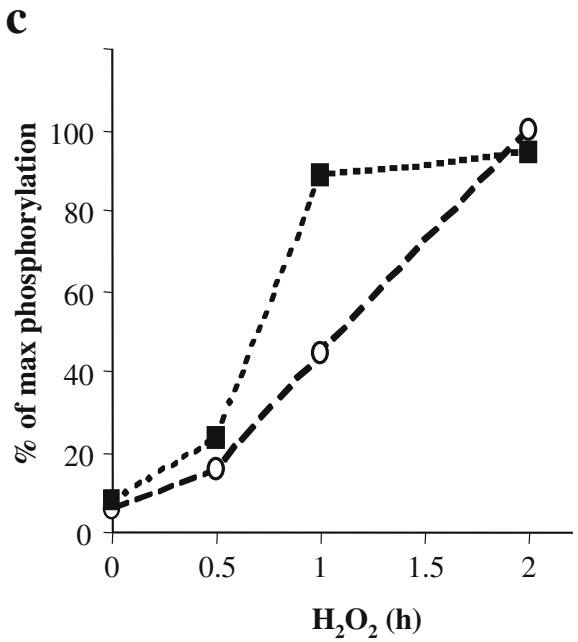

Ser632 phosphorylation almost completely in response to oxidative stress. The role of salicylic acid and SP600125insensitive kinases in total IRS1 phosphorylation in response to oxidative stress can be deduced from Fig. 6d, depicting marked but incomplete restoration of normal IRS1 migration in the gel. Importantly, the combination of salicylic acid with
SP600125 was more efficient than either individual inhibitor alone in preventing the retarded gel migration of IRS1.

Finally, the ability of combined treatment with salicylic acid and SP600125 to relieve insulin resistance at the level of $\mathrm{PKB} /$ Akt activation and at a metabolic level was studied. Insulin-stimulated phosphorylation of $\mathrm{PKB} / \mathrm{Akt}$ was signifi- a

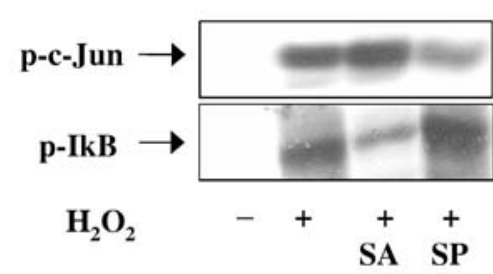

b

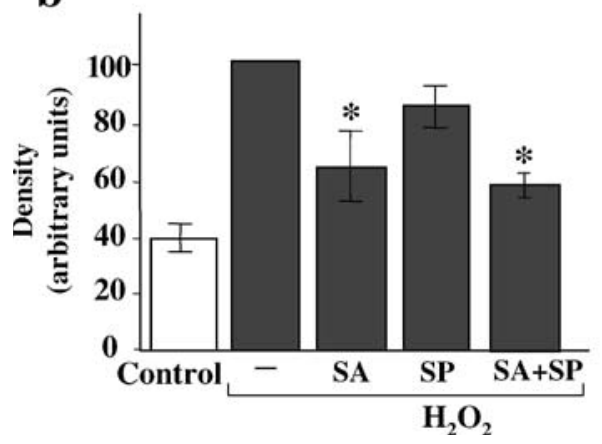

Fig. 6 Effects of SP600125 and salicylate on Ser632 and Ser307 phosphorylation of IRS1. Fao cells were preincubated for $60 \mathrm{~min}$ with $10 \mathrm{mmol} / 1$ salicylate (SA) and/or for $30 \mathrm{~min}$ with $40 \mu \mathrm{mol} / 1 \mathrm{SP} 600125$ (SP), before and during $2 \mathrm{~h}$ of exposure to oxidative stress $\left(\mathrm{H}_{2} \mathrm{O}_{2}\right)$. Cells were washed, lysed and subjected to western blot analysis. a Representative blots exposed to anti-phospho-c-Jun and anti-pIkB. Blots were exposed to $\mathbf{b}$ anti-pSer307-IRS1 or $\mathbf{c}$ anti-pSer632-IRS1, followed by c

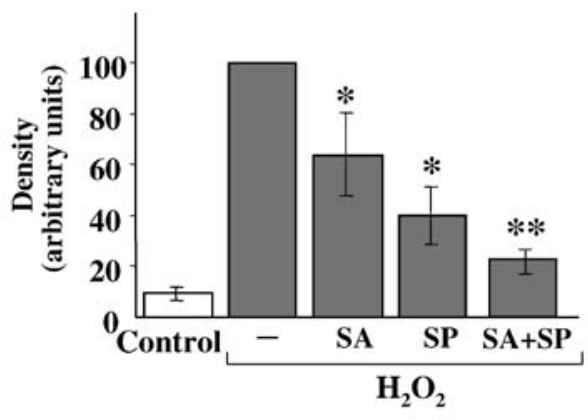

d

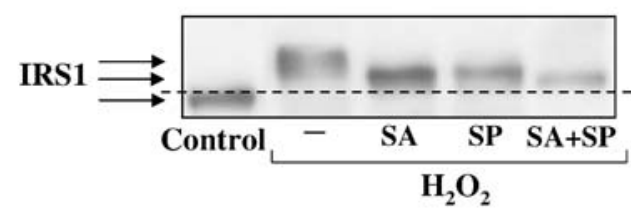

densitometry analysis. Results are mean \pm SEM of values derived from seven independent experiments yielding similar results, in which a value of $100 \%$ was assigned to the band intensity in oxidised cells. ${ }^{*} p<0.05$ and $* * p<0.01$ vs oxidised cells. A representative blot of membrane exposed to anti-IRS1 antibody is shown in $\mathbf{d}$. The dashed line denotes the gel migration of the IRS1 band in non-oxidised cells 
cantly increased by the combined use of salicylic acid with SP600125, amounting to over $50 \%$ of the response in control cells (Fig. 7a). To correlate these findings to a metabolic endpoint, we measured insulin-stimulated glycogen synthesis (Fig. 7b), demonstrating that the combined treatment of the cells with salicylic acid and SP600125 resulted in significant $(p<0.05)$ prevention of the impairment of insulin-induced glycogen synthesis induced by oxidative stress. These results suggest the capacity of combined stress-signalling inhibition with salicylic acid and SP600125 to protect against the induction of insulin resistance by oxidative stress.

\section{Discussion}

The present study suggests that phosphorylation of IRS1 on Ser307 or on Ser632 in response to oxidative stress defines two minimally overlapping pools of IRS1 molecules that are distinct spatially and functionally. The small pool of IRS1 molecules phosphorylated on Ser307 responds poorly to insulin and fails to be released from internal membranes. Its participation in mediating insulin resistance in response to oxidative stress is further supported by the finding that a combination of pharmacological stress kinase inhibitors can decrease Ser307 phosphorylation of IRS1 vis à vis partial restoration of insulin-stimulated $\mathrm{PKB} / \mathrm{Akt}$ phosphorylation and glycogen synthesis.

It is over a decade since the initial demonstrations that phosphorylation of IRS on Ser/Thr residues modulates its ability to undergo tyrosine phosphorylation and hence to function as a key mediator in propagating insulin receptor signalling [3]. Our understanding of the complexity of this regulatory mode has been steadily increasing. IRS polypeptides have dozens of Ser/Thr residues in their primary sequence, which are potential substrates for various Ser/Thr kinases. Some of these are kinases that are also intrinsic to the insulin signalling cascade, such as PKB/Akt, mTOR and $\mathrm{PKC}_{\zeta}$. These can mediate the physiological feedforward and shutdown mechanisms of the insulin signal [5], but additionally can act as modulators of insulin signal transduction when activated by other stimuli and/or extracellular cues. Other kinases not traditionally viewed as an integral part of the insulin signalling cascade, such as the stress kinases JNK1/2 and IKK $\beta$, mediate crosstalk between stress signalling and the insulin signalling cascade [1]. In particular, phosphorylation of IRS by these kinases tends to decrease insulin signalling efficiency and thus generate a state of insulin resistance. Further understanding of the role of Ser phosphorylation of IRS molecules will require the assignment of specific IRS Ser residues to biological outcomes, and identification of the IRS1 kinases responsible. For example, in cell lines treated with inducers of insulin resistance, including TNF- $\alpha$, chronic insulin and phorbol 12-myristate-13-acetate (PMA), it was suggested that pSer307-IRS1 mediates insulin resistance, but the kinases implicated in IRS1 phosphorylation were JNK1, $\operatorname{IKK} \beta$ or mTOR $[6,12,36]$. Nevertheless, consistent with its inhibitory role in insulin signal propagation, pSer307- $\mathbf{a}$

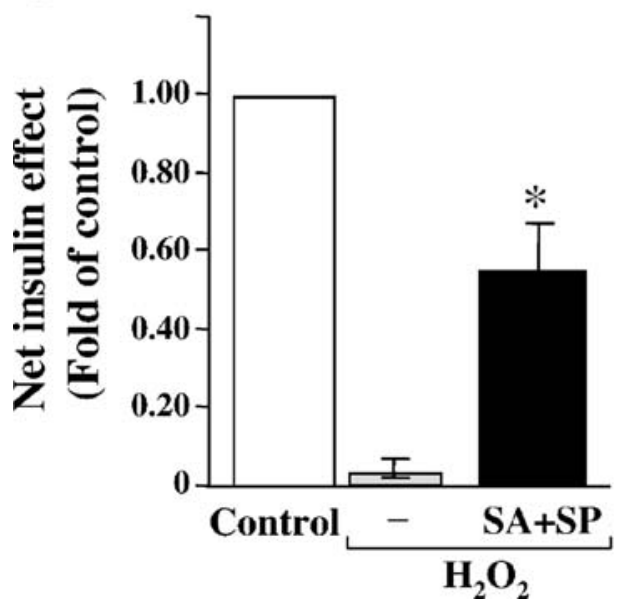

Fig. 7 Combined incubation with SP600125 and salicylate rescues oxidised cells from insulin resistance. a Fao cells were preincubated for $60 \mathrm{~min}$ with $10 \mathrm{mmol} / 1$ salicylate (SA) and for $30 \mathrm{~min}$ with $40 \mu \mathrm{mol} / 1 \mathrm{SP} 600125$ (SP), and were then exposed to oxidative stress $\left(\mathrm{H}_{2} \mathrm{O}_{2}\right)$ in the absence or presence of the two inhibitors for $2 \mathrm{~h}$. Cells were then washed and further incubated for $10 \mathrm{~min}$ in the absence or presence of $100 \mathrm{nmol} / 1$ insulin. The cells were washed, lysed and subjected to western blot analysis using anti-pSer473-PKB/Akt antibodies. Results are shown of densitometry analysis of seven b

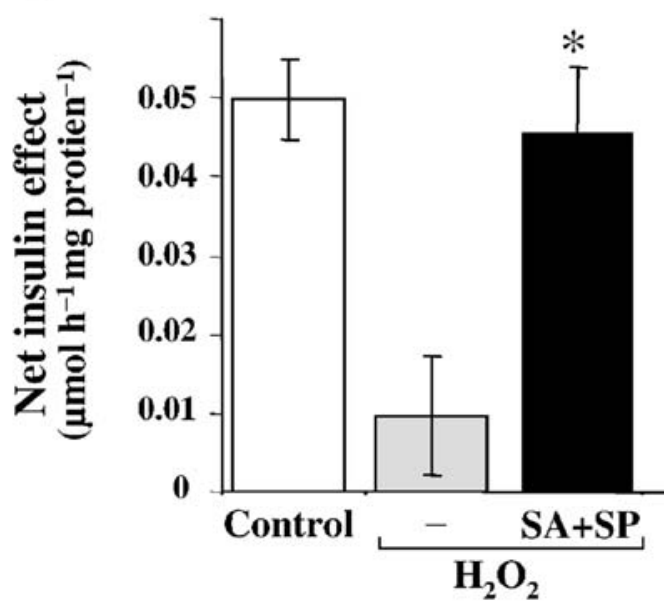

independent experiments in which a value of 1 was assigned to the net insulin effect on PKB/Akt phosphorylation in control cells. b Fao cells were treated with inhibitors under the oxidation conditions described above, followed by incubation for $60 \mathrm{~min}$ without or with $100 \mathrm{nmol} / 1$ insulin, during which glycogen synthesis was measured as described in Materials and methods. Results are the mean $\pm \mathrm{SEM}$ of the net insulin effect in five independent experiments. ${ }^{*} p<0.05$ vs the net insulin effect in oxidised cells 
IRS1 was increased in muscle of patients with type 2 diabetes and in insulin-resistant patients [13, 37].

The functional consequence of IRS1 phosphorylation on Ser632 is variable and may depend on the stimulus that triggers it. Phosphorylation of this site by rho kinase is required for normal transmission of the insulin signal [38]. In contrast, however, Ser 632 phosphorylation was suggested to play a negative role in signal propagation: it is increased in obesity in muscle of patients with type 2 diabetes and their insulin-resistant offspring, as well as after TNF administration [16-18]. In addition, it was demonstrated recently that nutrients can induce the phosphorylation of this site, and this phosphorylation negatively regulates the association between IRS1 and the PI3K [39].

Our results demonstrate an inhibitory role of pSer307IRS1 in the insulin signal in response to oxidative stress, as these molecules exhibited diminished insulin-induced tyrosine phosphorylation, interaction with $\mathrm{p} 85$, and associated PI3K activity (Fig. 4b,c). They also failed to be released from a membrane fraction in response to acute insulin stimulation (Fig. 4a). In contrast, pSer632-IRS1 seemed to participate normally in insulin signal propagation after oxidative stress. The finding that the two phosphoserine sites also define distinct cellular locations may be significant: various studies using biochemical and cell imaging approaches demonstrated that, in response to insulin stimulation, IRS1 is mobilised between cellular compartments and domains. Tyrosinephosphorylated IRS1 molecules may specifically accumulate in low-density microsomal fractions [30, 34, 35], potentially representing F-actin-rich sites in which they may serve to dock and activate PI3K $[32,34]$. Activating PI3K in such defined locations may be a specific effect of insulin, since it does not seem to occur in response to other stimuli, such as platelet-derived growth factor [33, 34, 40]. We and others have suggested that this unique ability of insulin to mobilise IRS1 and target PI3K may be impaired in response to oxidative stress, resulting in decreased activation of downstream signalling mediators and metabolic end-points [30, $33,41]$. Since the majority of IRS1 molecules were phosphorylated on Ser632, it is not surprising that insulinstimulated tyrosine phosphorylation and interaction with PI3K assessed in total cell lysates was normal after oxidative stress. However, pSer307-IRS1 molecules are retained in response to insulin stimulation in an internal membrane pool and have poor ability to propagate the insulin signal to $\mathrm{PKB} /$ Akt and to metabolic outcomes. The combination of spatial and functional characteristics of this small pool of IRS1 molecules renders them critical for the mechanism of insulin resistance in response to oxidative stress. Assigning such a potent function to a rather small fraction of IRS1 molecules is consistent with previous estimates that even under normal conditions insulin engages only a low percentage of the entire cellular IRS1 pool [35].
With the worldwide surge in the incidence of type 2 diabetes, there is a pressing need to discover new therapeutic approaches to the treatment of insulin resistance. The stress kinases link to Ser/Thr phosphorylation of IRS molecules is a molecular mechanism mediating the association between inflammation, endoplasmic reticulum stress, oxidative stress and insulin resistance, providing an attractive point for novel therapeutic intervention. Salicylates at high doses are known to improve glucose intolerance clinically, and have been shown to do so at least in part by inhibiting IKK $\beta$-mediated phosphorylation of IRS1 [42]. Pharmacological inhibitors of other stress kinases, including JNK, are being developed and are at various stages of testing for clinical use. Here we show that significant prevention of signalling and metabolic insulin resistance induced by oxidative stress can be achieved by the combined action of salicylates and SP600125 (Fig. 7). Stress sensing involves the activation of several parallel and interrelated signalling cascades, and rarely activates single proteins with IRS1 Ser/Thr kinase activity. The results of this study suggest that combined pharmacological inhibition of such kinases, rather than an attempt to target an individual stress kinase, may hold promise in relieving insulin resistance.

Acknowledgements This project was supported in part by grants from D Cure-Diabetes Care in Israel (to N. Bashan and A. Rudich), by INSERM (to J. F. Tanti, P. Gual Y. Le Marchand-Brustel) and by the National Institute of Biotechnology Negev, BGU, Israel (to A. Rudich and N. Bashan). We thank A. Klip for helpful discussion and T. Tarnovscky for excellent technical help.

\section{References}

1. Evans JL, Goldfine ID, Maddux BA, Grodsky GM (2002) Oxidative stress and stress-activated signaling pathways: a unifying hypothesis of type 2 diabetes. Endocr Rev 23:599-622

2. Evans JL, Goldfine ID, Maddux BA, Grodsky GM (2003) Are oxidative stress-activated signaling pathways mediators of insulin resistance and beta-cell dysfunction? Diabetes 52:1-8

3. Zick Y (2005) Ser/Thr phosphorylation of IRS proteins: a molecular basis for insulin resistance. Sci STKE 2005:pe4

4. Tanti JF, Gual P, Gremeaux T, Gonzalez T, Barres R, Le Marchand-Brustel Y (2004) Alteration in insulin action: role of IRS-1 serine phosphorylation in the retroregulation of insulin signalling. Ann Endocrinol (Paris) 65:43-48

5. Gual P, Le Marchand-Brustel Y, Tanti JF (2005) Positive and negative regulation of insulin signaling through IRS-1 phosphorylation. Biochimie 87:99-109

6. Gual P, Gremeaux T, Gonzalez T, Le Marchand-Brustel Y, Tanti JF (2003) MAP kinases and mTOR mediate insulin-induced phosphorylation of insulin receptor substrate-1 on serine residues 307, 612 and 632. Diabetologia 46:1532-1542

7. Gual P, Le Marchand-Brustel Y, Tanti J (2003) Positive and negative regulation of glucose uptake by hyperosmotic stress. Diabetes Metab 29:566-575

8. Gual P, Gonzalez T, Gremeaux T, Barres R, Le Marchand-Brustel Y, Tanti JF (2003) Hyperosmotic stress inhibits insulin receptor 
substrate-1 function by distinct mechanisms in 3T3-L1 adipocytes. J Biol Chem 278:26550-26557

9. Hiratani K, Haruta T, Tani A, Kawahara J, Usui I, Kobayashi M (2005) Roles of mTOR and JNK in serine phosphorylation, translocation, and degradation of IRS-1. Biochem Biophys Res Commun 335:836-842

10. Gao Z, Hwang D, Bataille F et al (2002) Serine phosphorylation of insulin receptor substrate 1 by inhibitor kappa B kinase complex. J Biol Chem 277:48115-48121

11. Gao Z, Zuberi A, Quon MJ, Dong Z, Ye J (2003) Aspirin inhibits serine phosphorylation of insulin receptor substrate 1 in tumor necrosis factor-treated cells through targeting multiple serine kinases. J Biol Chem 278:24944-24950

12. Aguirre V, Uchida T, Yenush L, Davis R, White MF (2000) The c-Jun NH2-terminal kinase promotes insulin resistance during association with insulin receptor substrate-1 and phosphorylation of Ser307. J Biol Chem 275:9047-9054

13. Bandyopadhyay GK, Yu JG, Ofrecio J, Olefsky JM (2005) Increased p85/55/50 expression and decreased phosphatidylinositol 3-kinase activity in insulin-resistant human skeletal muscle. Diabetes 54:2351-2359

14. Mussig K, Staiger H, Fiedler H et al (2005) Shp2 is required for protein kinase $\mathrm{C}$-dependent phosphorylation of serine 307 in insulin receptor substrate-1. J Biol Chem 280:32693-32699

15. Werner ED, Lee J, Hansen L, Yuan M, Shoelson SE (2004) Insulin resistance due to phosphorylation of insulin receptor substrate 1 at serine 302. J Biol Chem 279:35298-35305

16. Morino K, Petersen KF, Dufour S et al (2005) Reduced mitochondrial density and increased IRS-1 serine phosphorylation in muscle of insulin-resistant offspring of type 2 diabetic parents. J Clin Invest 115:3587-3593

17. Bouzakri K, Roques M, Gual P et al (2003) Reduced activation of phosphatidylinositol-3 kinase and increased serine 636 phosphorylation of insulin receptor substrate-1 in primary culture of skeletal muscle cells from patients with type 2 diabetes. Diabetes 52:1319-1325

18. Plomgaard P, Bouzakri K, Krogh-Madsen R, Mittendorfer B, Zierath JR, Pedersen BK (2005) Tumor necrosis factor-\{alpha\} induces skeletal muscle insulin resistance in healthy human subjects via inhibition of Akt substrate 160 phosphorylation. Diabetes 54:2939-2945

19. Kaneto H, Nakatani Y, Kawamori D et al (2005) Role of oxidative stress, endoplasmic reticulum stress, and c-Jun N-terminal kinase in pancreatic b-cell dysfunction and insulin resistance. Int $\mathrm{J}$ Biochem Cell Biol 37:1595-1608

20. Haber CA, Lam TK, Yu Z et al (2003) $N$-acetylcysteine (NAC) and taurine prevent hyperglycemia-induced insulin resistance in vivo: possible role of oxidative stress. Am J Physiol Endocrinol Metab 285:E744-E753

21. Rudich A, Bashan N (2002) Cellular redox state and insulin sensitivity: potential role of lipoic acid. In: Zierath JR, WallbergHenriksen H (eds) Muscle metabolism. Taylor and Francis, London, pp 329-348

22. Robertson G, Leclercq I, Farrell GC (2001) Non-alcoholic steatosis and steatohepatitis. II. Cytochrome P-450 enzymes and oxidative stress. Am J Physiol (Gastrointest Liver Physiol) 281:G1135-G1139

23. Maddux BA, See W, Lawrence JC Jr, Goldfine AL, Goldfine ID, Evans JL (2001) Protection against oxidative stress-induced insulin resistance in rat L6 muscle cells by micromolar concentrations of alpha-lipoic acid. Diabetes 50:404-410

24. Rudich A, Kozlovsky N, Potashnik R, Bahan N (1997) Oxidant stress reduces insulin responsiveness in 3T3-L1 adipocytes. Am J Physiol (Endocrinol Metab 35) 272:E935-E940

25. Hansen LL, Ikeda Y, Olsen GS, Busch AK, Mosthaf L (1999) Insulin signaling is inhibited by micromolar concentrations of $\mathrm{H}_{2} \mathrm{O}_{2}$. Evidence for a role of $\mathrm{H}_{2} \mathrm{O}_{2}$ in tumor necrosis factor alphamediated insulin resistance. J Biol Chem 274:25078-25084
26. Houstis N, Rosen ED, Lander ES (2006) Reactive oxygen species have a causal role in multiple forms of insulin resistance. Nature 440:944-948

27. Potashnik R, Bloch-Damti A, Bashan N, Rudich A (2003) IRS1 degradation and increased serine phosphorylation cannot predict the degree of metabolic insulin resistance induced by oxidative stress. Diabetologia 46:639-648

28. Rudich A, Tirosh A, Potashnik R, Hemi R, Kanety H, Bashan N (1998) Prolonged oxidative stress impairs insulin-induced GLUT4 translocation in 3T3-L1 adipocytes. Diabetes 47:1562-1569

29. Kozlovsky N, Rudich A, Potashnik R, Bashan N (1997) Reactive oxygen species activate glucose transport in L6 myotubes. Free Radic Biol Med 23:859-869

30. Tirosh A, Potashnik R, Bashan N, Rudich A (1999) Oxidative stress disrupts insulin-induced cellular redistribution of insulin receptor substrate-1 and phosphatidylinositol 3-kinase in 3T3-L1 adipocytes. J Biol Chem 274:10595-10602

31. Hadari YR, Tzahar E, Nadiv O et al (1992) Insulin and insulinomimetic agents induce activation of phosphatidylinositol 3 '-kinase upon its association with pp185 (IRS-1) in intact rat livers. J Biol Chem 267:17483-17486

32. Patel N, Rudich A, Khayat ZA, Garg R, Klip A (2003) Intracellular segregation of phosphatidylinositol-3,4,5-trisphosphate by insulin-dependent actin remodeling in L6 skeletal muscle cells. Mol Cell Biol 23:4611-4626

33. Tirosh A, Rudich A, Potashnik R, Bashan N (2001) Oxidative stress impairs insulin but not platelet-derived growth factor signalling in 3T3-L1 adipocytes. Biochem J 355:757-763

34. Clark SF, Martin S, Carozzi AJ, Hill MM, James DE (1998) Intracellular localization of phosphatidylinositide 3-kinase and insulin receptor substrate-1 in adipocytes: potential involvement of a membrane skeleton. J Cell Biol 140:1211-1225

35. Inoue G, Cheatham B, Emkey R, Kahn CR (1998) Dynamics of insulin signaling in 3T3-L1 adipocytes. Differential compartmentalization and trafficking of insulin receptor substrate (IRS)-1 and IRS-2. J Biol Chem 273:11548-11555

36. Jiang G, Dallas-Yang Q, Liu F, Moller DE, Zhang BB (2003) Salicylic acid reverses phorbol 12-myristate-13-acetate (PMA)and tumor necrosis factor alpha (TNFalpha)-induced insulin receptor substrate 1 (IRS1) serine 307 phosphorylation and insulin resistance in human embryonic kidney 293 (HEK293) cells. J Biol Chem 278:180-186

37. Corbould A, Kim Y-B, Youngren JF et al (2005) Insulin resistance in the skeletal muscle of women with PCOS involves intrinsic and acquired defects in insulin signaling. Am J Physiol Endocrinol Metab 288:E1047-E1054

38. Furukawa N, Ongusaha P, Jahng WJ et al (2005) Role of rho-kinase in regulation of insulin action and glucose homeostasis. Cell Metab 2:119-129

39. Tzatsos A, Kandror KV (2006) Nutrients suppress phosphatidylinositol 3-kinase/Akt signaling via raptor-dependent motor-mediated insulin receptor substrate 1 phosphorylation. Mol Cell Biol 26:63-76

40. Ricort JM, Tanti JF, Van Obberghen E, Le Marchand-Brustel Y (1996) Different effects of insulin and platelet-derived growth factor on phosphatidylinositol 3-kinase at the subcellular level in 3T3-L1 adipocytes. A possible explanation for their specific effects on glucose transport. Eur J Biochem 239:17-22

41. Ogihara T, Asano $T$, Katagiri $\mathrm{H}$ et al (2004) Oxidative stress induces insulin resistance by activating the nuclear factor-kappa B pathway and disrupting normal subcellular distribution of phosphatidylinositol 3-kinase. Diabetologia 47:794-805

42. Kim JK, Kim YJ, Fillmore JJ et al (2001) Prevention of fatinduced insulin resistance by salicylate. J Clin Invest 108: $437-446$ 